\title{
Perceptions of health care professionals on Governance of Hospital Quality of Care and Accreditation in the Public Hospitals of United Arab Emirates: A Qualitative Study
}

Ghada Ali ( $\sim$ farahghada2@gmail.com )

Research article

Keywords: Accreditation, healthcare quality, hospital management, Governance, UAE, Ministry of Health and Prevention, MOHAP

Posted Date: November 9th, 2019

DOI: https://doi.org/10.21203/rs.2.16963/v1

License: (9) This work is licensed under a Creative Commons Attribution 4.0 International License.

Read Full License 


\section{Abstract}

Background Hospital accreditation is frequently used as a tool for government directives for the purpose of guaranteeing patient safety or quality of care. The study aims to interview healthcare workers with a diversity of views and perceptions on accreditation experiences, performance and governance of quality of care.

Methods: Semi-Structured interviews between June 2017 - Dec 2017 with 155 board members and executives from four public acute care accredited MOHAP hospitals in the United Arab Emirates was undertaken. The interviews mainly highlighted the role played by the management in overseeing the quality of care in their respective hospitals. Perceptions of interviewees' regarding factors that have influenced their present approach to governance in this area were also elicited. Thematic analysis was utilized for the purpose of identifying the major themes extracted from the interview transcripts.

Results A total of four themes emerged from the analysis including (1) Corporate (Hospital) Governance, (2) Accreditation, (3) Employees' satisfaction, (4) Quality Management and Performance. Interviews with United Arab Emirates health care leaders emphasized on the governance role and affirmed that they had a well-structured governing body. Furthermore, the role of the leadership at their hospital was very essential in making the accreditation happen and in improving the quality of care provided to patients. Staff expressed strong support for implementation as well as development of hospital standards along with requesting for ongoing motivation and recognition. Respondents also cited the need for infrastructure, funding and technical assistance as the major obstacles.

Conclusion The study demonstrated a strong support for the implementation or development of hospital standards. Implementing quality improvement programs such as accreditation leads to enhanced quality of care provided at the hospitals and better health outcomes. This study emphasizes the importance of having effective governance, top management commitment and leadership side by side for effective implementation of accreditation. Findings shows that strong governance and support from the top management were critical for implementing accreditation and improving quality of services in the government hospitals.

\section{Introduction}

Public hospitals accounts for most of the acute care provision in the United Arab Emirates and it considerably plays a vital role in the health system of the nation. Growing concerns over patient safety in the hospitals and public accountability provoked the overview of different regulatory, reporting requirements, and quality improvement programs. Accreditation is an external process review intended to evaluate how staff accomplish their goals in relation to established standards. These types of programs exist in several different forms and are largely meant to direct an organization towards meeting societal objectives ${ }^{1}$. An external, particularly trained group broadcast a survey to evaluate compliance with predefined standards. The major objective of accreditation is to ensure and stimulate safe and high- 
quality care. Such goals are often accomplished based on both the nature of the controls as well as how well an organization becomes regulated to answer the controls confined within a certain regulatory system. Programs of accreditation are purely regulatory control systems that aims to examine the performance of organization against explicit standards $s^{2-5}$. In several other countries, the programs of accreditation represent an endorsement for the provision of quality services ${ }^{6,7}$.

Improving the hospital care quality often helps to achieve the health-related sustainable development goals. However, there are several factors that may impact the quality of hospital care such as health workforce shortages, weak governance and management, malfunctioning equipment, pharmaceutical shortages, quality improvement programs deprivation, inadequate diagnostic capabilities, record-keeping, and poor coordination of care with other facilities. Braithwaite ${ }^{8}$ have argued that the empirical evidence for sustaining different claims regarding advantages of accreditations is also lacking at present. Several countries including United Arab Emirates have frequently used accreditation as a tool for government regulation to improve patient safety and guarantee quality of care.

Implementing accreditation standards is purely demanding for different organizations and individuals ${ }^{9}$. Furthermore, empirical and theoretical evidence on accreditation is also lacking mainly in the emerging economy of Middle East. Research studies conducted previously have shown inconsistent results with respect to the impacts of accreditation. Consequently, there is an extensive call in health care evidence to assess the external systems for accreditations to fabricate rigorous assessments of their impact ${ }^{10}$.

Some major concerns that may impact the staff retention and satisfaction in public health hospitals are therefore a matter of apprehension. The foundational influences on the relationship between staff and manager are located under governance domain. Fukuyama ${ }^{11}$ demonstrated good governance as requiring suitable levels of agent such as staff autonomy for making decision to be ascertained. Therefore, it is critical for a manager to make correct decisions and judgments regarding their staff work and motivation. Regulators often aim to encourage the implementation of change programs in the healthcare organizations designed to improve financial or clinical performance. Despite the widespread use of accreditation in many countries, and common belief that it contributes to improvements in organizational outcomes, there is limited scholarly research that establishes or explores this relationship ${ }^{12}$. Moreover, there has been an emerging evidence with respect to the governance and transformation of the healthcare system, however, a substantial gap in literature exist regarding how some of the specific tools or governance mechanisms work, in what situations, and how health system actors gets impacted- predominantly health workforce. Establishing the mechanisms of governance and outcomes for health workforce, can better help decision makers to develop plans and future initiatives.

Ministry of Health and Prevention (MOHAP) at the UAE has launched its accreditation program in 2012 to improve quality across the continuum of care. Presently, MOHAP has achieved the international accreditation for $\mathbf{4 9}$ facilities from both the "Joint commission International Accreditation and accreditation Canada." National strategy of the United Arab Emirates aims to raise the quality of healthcare services to best international practice levels by 2021 in order to keep up with abrupt scientific 
progress in both the managerial and medical aspects for incessantly improving the quality together with

offering an integrated healthcare service ${ }^{13}$. Furthermore, patients often seeks for superior quality services while, employees hunt for additional scientific or managerial services. For this reason, the significance of implementing the quality programs and initiatives for example, accreditation and governance arises because of the need to improve quality in health services, maintain the rights for both patients and employees, as well as meet and exceeds the expectations of its customers. Therefore, this paper seeks to address the views and perceptions of hospital management, and frontline employees from four public acute care hospitals about governance concerning quality of care generally and hospital accreditation experiences and employee performance particularly.

\section{Methods}

\section{Study design}

We used a qualitative study design using in-depth interviews with the employees in four public acute care accredited MOHAP hospitals in the United Arab Emirates.

\section{Settings}

The study was performed in four acute care hospitals. The Ministry of Health and Prevention (MOHAP) directs 17 hospitals with around 2,127 acute and long stay hospital beds that delivers the vast majority of the country's emergency and elective outpatient or inpatient care. All of these hospitals offer vastly specialized healthcare and serve more than 60,000 patients a month in their Accident and Emergency Units. They serve more than 6,500 inpatients and 70,000 outpatients a month.

The selected hospitals are acute, general hospitals that offers secondary services. They have a bed capacity of 70 to 150 beds, and around 1690 employees. The hospitals also offers core and specialized services for both non-nationals and nationals in the Northern emirates in addition to accept referrals from other emirates in the UAE, either private or government hospitals. Consequently, for our study, any JCIA accredited acute care hospital operative under the MOHAP in the UAE and having received JCIA in the period between 2013 and 2017 was entitled for the study. This range of years fits the study period. By 2018, eight hospitals have secured international accreditation.

\section{Participants}

Out of the 17 hospitals, four on purpose (purposive sampling) were selected for the study, as they were the only hospitals accredited at the time of conducting the study, and they met the inclusion criteria, after 
purposive sampling procedure and all of the possible respondents in these four hospitals (healthcare professionals and administrators/managers) were questioned. Study included 155 healthcare professionals. These professionals were approached to assess their willingness to participate in key informant interviews on governance in public hospitals. These participants were included because they were acquainted with the standards use and had also contributed in an earlier UAE national accreditation program.

\section{Data collection procedure and tools}

Data was collected by the investigator directly from personal or real-life experience i.e. semi structured indepth interviews. This method for data collection was employed to acquire adequate knowledge based on the perceptions and experiences of the participants. The semi-structured interview approach involved the preparation of an interview guide that included a predetermined list of questions. The interview guide served as a checklist to ensure that the same questions were asked to all participants but at the same time had a great deal of flexibility. Structured open-ended questions supplemented with probing were used to elucidate deeper reflections and opinions of the participants on a one-to-one basis along with establishing an enhanced relationship with them to enable data provision. Persistent interviews were conducted until data saturation was achieved. Extensive notes were also jotted down during interviews that were utilized subsequently to extract themes and build an inventory of governance related behaviors or practices that participants regarded as being associated with management effectiveness in the public hospitals.

\section{Data analysis}

The data acquired from the interviews were recorded, coded, transcribed and analyzed to identify a set of key themes from the opinions articulated. The interviews were digitally voice recorded after securing interviewee consent. Interviews were conducted in both Arabic and English based on the respondent and were transcribed verbatim immediately thereafter. Analysis of the transcriptions from 155 respondents identified four broad themes using thematic analysis. Throughout this process, the lead investigator regularly met with "research advisory group" to confer the developing framework being applied to infer the data.

\section{Ethical approval}


Ethical approval to conduct this study was sought and obtained from the relevant committees at both the academic and governmental levels including Hamdan Bin Mohammed Smart University $(\mathrm{HbMsU})$ and the Medical Research Ethics Committee at the Ministry of Health and Prevention (MOHAP) at the United Arab Emirates (UAE). Interviewees were assured of anonymity and each gave written consent to participate. The data was preserved in the password encrypted laptop of the investigator. The data was only available to the researcher due to the principles of confidentiality and secrecy of the respondents. This data was gathered without attached names, and audio recordings were deleted after transcription was completed.

\section{Results}

Findings of the study are organized below based on the themes that arose during the interviews. Direct quotes are used to exemplify and support points. Where compulsory, some minor particulars have been altered to preserve the interviewee's anonymity.

\section{Corporate (Hospital) Governance}

All hospital directors and their deputies discussed their experience with changing the system at their hospitals and strategies in order to facilitate change necessary for new accreditation standards to be implemented. Embedding the new standards and practices into the existing systems was considered the most efficient way to sustain practices and the most cited approach used by the executive leaders. The first measure assured included well-structured governing body and their role, along with clear mission, vision, strategic and operation plans in line. Moreover, most of the respondents agreed that the executive committee and the executive leaders played a major role in the accreditation process and in their interaction with the hospital staff in establishing the hospital quality strategy. One of the hospitals' directors stated that,

"(It) enhanced my reputation among other directors".

Another hospital director stated;

"Accreditation strengthened the relationship between the top management and the employees."

When they were asked about who exerted the greatest impact and helped in accreditation and in Quality Improvement (QI) in the hospital, majority of the respondents said that with the support of the executive committee while, the top management named the head of quality department had the greatest impact. When the middle management, line managers, and employees were asked about who exerted the greatest impact and helped in accreditation and on QI in the hospital, most of the respondents named the 
executive committee and the top management as having the greatest impact. Yet, some of the respondents named the executive in charge of quality as having the greatest impact. One staff specified,

"It's really important the members of the executive committee are engaged in a real way and supported the process of accreditation and smoothened all the challenges faced."

One of the executive committee members stated,

"Our hospital director is making sure that patients' safety issues are put on the agenda first, so she's also a very good driving force for it."

Across all the employees interviewed, participants indicated their satisfaction with the hospital management, particularly in decision-making, and on-the-job training. The following narratives emphasize this:

"Our hospital management involve us in decision-making." We had departmental meetings where we provide our views. "We also have on-the-job training sessions - all staff are undergoing health information system training".

\section{Accreditation}

Most of the respondents affirmed that accreditation led to improvement in the quality of services provided in several areas; for example, implementing standards, policies and procedures and rules and regulations, improving documentation, introducing quality programs and initiatives. Another frequently mentioned benefit of accreditation was to make sound decisions based on facts through the adoption of key performance indicators, which are measured frequently and compared to other hospitals' findings nationally and internationally. Translating the improvements in the patients' satisfaction and enhancing their trust in the hospital. Other mentioned benefits of accreditation included: strengthened relationships between the hospitals departments and other hospitals as stated by one of the heads of departments;

"If a national program needs to be adopted to improve patients' safety or to improve the communication between staff, accreditation can be the good choice for this. It's a tool for changing and a system of improvement in all aspects such as patient safety, quality of health care services".

One of the respondents saw accreditation as an effective tool for enhancing safety, as she stated that

"Accreditation is a tool improving the quality of work in my hospital. It was a big job that we had done at that time and it meant a lot for us and my team in the hospital."

Another head of department viewed accreditation as a safety tool or as a process to enhance safety, other employee saw it as a tool used to measure the quality of services, with a view to improving the services. 
"It's the measurement tool for quality and safety, so it's a tool to initiate the culture of safety and quality".

Other interviewees believed that accreditation is a valuable quality improvement tool as compared to the previous initiatives that were used by the Ministry of Health and prevention as improvement plans. This reflected a favorable attitude towards accreditation, with a belief that this was capable to promote quality improvement than other past initiatives.

Another quote which reflected a positive attitude towards accreditation was by a hospital director who claimed:

"I think it is a way for a reality improvement. It's a valuable tool to improve the quality of the health care system. It's a guidance in all aspects."

The commitment and support from management, establishing an accreditation committee to guide implementation, distributing tasks, and effective teamwork, as well as supporting the continuous training and workshops were reported as key for implementing a smoother accreditation process. Employees' interest and motivation toward accreditation as it was the first time for them to go into such experience was one of the enabling factors. At the same time, employees as well as hospitals' top management reported facing many challenges in implementing the requirements of the accreditation and getting the accreditation. Limited financial resources and inadequate staff to carry out the workload of accreditation were the main challenge impeding the implementation of the standards. For example, financial resources were needed for infrastructure, equipment and IT, and for staff.

Staff initially perceived accreditation as a vague process and were worried about the preparation for accreditation, about the increased amount of workload, and being surveyed. However, the extensive training and workshops provided and supported by the Ministry of Health and Prevention top management were successful in overcoming this challenge.

Resistance from staff, especially among older staff and physicians, was reported as a major challenge. One of the heads of departments in a surgical ward stated that

"at the beginning the concept was totally new for the stall and for all of us, to engage them and motivate them and make them believe in the process and the importance of it was quite challenging, I found resistance from them."

Some departments' directors stated that older staff members were more resistant to change. Others, however, thought there was no difference between the older and younger staff, and some suggested that younger staff were more challenging to engage with. Reasons included lack of motivation, fear of new change, and unwillingness to learn new system.

"Actually, we face many challenges when we meet with the staff and discuss progress in the preparation for the accreditation, like staff resistance because they have workload and they don't have time to work 
extra. Unfortunately, it is from the younger generations because they are maybe lazy; they do not want anything as extra work without any rewards. Rewards are a very important issue for them."

Another claimed: "I think the resistance was more from the older generations such as 45 to 55 years old. They were just not willing to change what they are used to, plus they took at it as extra work to be done."

The need for financial support was suggested repeatedly for improving the implementation of accreditation, as one hospital director illustrated

"Financial support was needed to improve the infrastructure of the hospital] for improving the fire and alarm system, providing the needed equipment."

Another suggestion was made by a quality coordinator:

"Appointing adequate and qualified employees were needed, carrying the extra workload, monitoring, supervising, and guiding the continuous implementation of improvements in order to maintain sustainability of the developed measures, and complete the development and implementation of all measures."

Conducting follow-up meetings, communication, and collaboration with other hospitals were suggested for sharing experiences on implementing accreditation. Some front-line employees suggested that training project managers and appointing local experts to train the staff could improve the delivery of services:

"One strategy to improve implementation is to establish a train the trainer program that includes experts from the UAE, from the different hospitals who are aware of the context of UAE-government hospitals] in order to attain the accreditation successfully and with minimal findings."

One of the hospitals directors reported,

"Adequate resources including the human resources is essential to handle the accreditation and improve the quality of services at our hospitals, especially with the added workload on all the staff."

\section{Employees' satisfaction}

The members of the executive committee headed by the hospital director and supported by MOHAP headquarters described activities that empowered, motivated, and reinforced staff involvement with the accreditation. Their actions to empower staff also included allowing them more power to authorize resources. Leadership walk rounds were considered as a particularly useful tool for shared dialogue and as a listening exercise to both patients and for speaking with frontline employees across the hospital. They further affirmed that the role of the governing body and top management were key in 
making the accreditation happen in providing support to employees, empowering them, and facilitating the provision of resources. As stated by one of the frontline employees

"we've got leadership rounds, and that's made a big difference to identifying the challenges on the wards and in solving it."

Since employees' engagement is an important pillar for the success of an accreditation program, thus, it was essential to ask the interviewees about their engagement in the accreditation process and understand the tasks they were involved in. The members of the executive committee described activities that empowered, motivated, and reinforced staff involvement with the accreditation. Old staff and doctors were the most resistant people; therefore, convincing them and facilitating their engagement and showing the benefit of accreditation to them on the personal and facility level was required. Their constant communication with staff was critical to encourage and ensure their engagement with the program. Regular meetings with different committees based on the accreditation standards offered direction and support by involvement from the hospital leaders.

One of the heads of departments described her engagements and role as

"I was the head of the Governance and leadership team of the JCIA".

She said about her engagement in the accreditation preparation;

"I am the leader in this chapter, and I run the leadership committee. Its aim is to ensure that the requirements of the standard is well implemented, I was responsible on organizing, distributing, delegating, observing, and following up, and trying to improve the professionalism and improving the communication skills."

Involvement and training of staff in the accreditation program helped in their motivation and engagement as stated by one of the front-line staff:

"In the beginning we faced a difficultly but later on when we were trained everything was settled, we became more interested in the accreditation process."

The top management and employees indicated that training and education was provided to staff to prepare them better for the accreditation process, which helped employees perceive accreditation as an opportunity for professional development and for providing high-quality services, as one of the employee mentioned,

"After training and understanding accreditation process and requirements, it changed my whole perception. It changed the way I interact with people. It changed the way I manage things. I mean, my whole perception was different."

Accreditation helped enhance communication and teamwork among staff and between staff and management staff and patients. As one of the hospital's director illustrated: 
"Patients started feeling that doctors and nurses are better communicating with them and explaining to them what they need to know. Patients became more aware of their rights and felt that the healthcare providers became more involved in their healthcare."

All the hospital directors and their deputies highlighted the importance of their commitment and most believed that they acted as a support to staff implementing the accreditation standards. Some of the examples of their commitment included: attending learning sessions, leadership walk rounds were considered as a particularly useful tool for ensuring the availability of safe culture, listening to staff and patients, integrations of safety into the executive committee meetings agenda such as occurrence variance Reports (OVRs) and sentinel events at meetings and prioritizing it on the agenda. In addition, all the hospital staff agreed that the hospitals' top management acted as role models to others and most agreed on the powerful effects that their visible commitment had. According the executive committees headed by the hospital's directors, many times the top management were called in to deal with the resistance of some staff.

Many of the staff interviewed stated that the hospitals top managements' commitments and involvement made a significant contribution to the success of the accreditation process in the hospitals. Another line manager stated,

"I certainly know that our hospital director and representative from MOHAP headquarters have met with all the heads of the committees in small groups. Certainly, and the hospital director said, if you've got problems come directly to me"

\section{Quality Management and Performance}

Monitoring the progress of the preparation for accreditation was a frequently reported activity by both hospital directors, middle, and line managers. The members of the executive committees' monitored progress by reviewing set goals and performance measures, reviewing the reports and asking some questions on some activities; in particular, patients' safety issues and challenges facing the effective implementations of $\mathrm{JCl}$ standards and discussing it at the executive committee meetings. Such outcomes were reviewed on a weekly or quarterly basis depending on the hospital.

Monitoring was not only to explore challenges, but also as a way of ensuring that targets were met. Regular meetings with different committees on the accreditation standards allowed joint oversight, offered direction and support, and was another provision of involvement and support from the hospital's leaders. Feedback from the committee to the senior management at the MOHAP level and the executive committee at hospital level on whether staff was complying with accreditation and standards prescribed activities was thought to be a powerful influence on staff engagement and accountability. This is because staff were influenced by positive or negative responses from senior management. 
For some of the interviewees, the use of data and its source to monitor the progress was important. However, there were different ideas on which data to use. For example, for one the staff, data had to come from both staff and patients:

"Measuring the outcome on both the consumer and the employee and see the positive outcomes resulting from this process."

On the other hand, for the Head of department, the patient view was the important issue "frequent meetings with the staff and Patients are essential to monitor for the progress."

\section{Discussion}

The findings of the study implies that accreditation is often associated with improvement of the healthcare quality, improvement in support services quality, and documentation. Improvement in quality may highly be reflected by the increase in the satisfaction of employee. All hospital directors, their deputies for medical, nursing and administrative affairs, in addition to the middle, line managers and front-line employees affirmed that they had a well-structured governing body with essential role. The proper functioning of the governing body, as per all respondents, required the collaboration of all employees in different categories in putting and making the organizations' mission and vision clear to everyone, putting the strategic and operation plan, facilitating the accreditation process, and in making sure that the employees are compliant with the standards, rules and regulations.

Accreditation was seen to improve the quality of services delivered, in particular through standardizing delivery of services, improving the local healthcare culture, improving teamwork and collaboration across the hospitals. Respondents agreed that professionals had a positive attitude towards the role of governing body and the top management in achieving the accreditation and fostering its impact. The results stressed on the fact that support from the management and strong leadership were crucial for improving quality of services in the hospitals. Moreover, staff training was also important to improve their overcoming resistance and experience. These finding are consistent with previous studies that have indicated that accreditation has positive impact on the hospital's quality performance. In a study carried

out by Schmaltz et al. ${ }^{14}$ in the USA on 3,891 hospitals, it was found that the likelihood that a hospital was a high performer in clinical quality measures between 2004 and 2008 was significantly associated with Joint Commission accreditation status. Another study explored the perceptions of nurses working at accredited hospitals in Lebanon. The findings suggested that hospital accreditation improved quality of care $^{15}$. Education and training of staff were critical for the implementation of accreditation ${ }^{15,16}$. Additionally, providing incentives, resources, rewards and publicizing the names of centers were considered effective marketing tools for the centers leading towards employee's satisfaction ${ }^{17}$.

Indeed, most of the scholars identified the financial resources as a barrier for implementing accreditation. Such barrier is found to be major as it affected several different aspects of the accreditation program, including staffing issues, information dissemination, and training ${ }^{16}$. The authors showed impediments in 
recruiting staff and equipment due to lack of financial resources. Another area that was highlighted in the interview included the staff reward. This was considered another essential need by the staff in order to be motivated and to take on the extra workload.

Staff shortage was another issue. The accreditation process requires sustainability; thus, staff shortage represents major barriers to successful implementation and its sustainability also noted by Ongori ${ }^{18}$, and Richman et al., ${ }^{19}$. The findings suggest that enthusiasm and universal support for adopting standards as well as working to improve the care quality and environment. The health worker's dedication in the four of the facilities to high-quality care of the patient was also evident. Upgrades were needed to improve patient care quality that would also improve working conditions for health professionals, thus, leading to improved morale, performance and job satisfaction.

This study is the first of its kind both nationally and internationally investigating the effect of corporate governance on accreditation and hospitals quality performance. However, the study had some limitations: the study only included four UAE government hospitals. The selection of only government hospitals makes it unclear how generalizable the findings are to the other hospitals such as the private ones. It is therefore, recommended that future research is replicated on a larger scale to include more government, private hospitals, and primary care institutions in order to get more generalizable and reliable understanding. Moreover, this study is based on the perception of the government hospitals employees and no feedback was taken from the patients. It is highly recommended that future research examines the impact of accreditation in conjunction with patient outcome measures from the patients' perception. The decision to include semi-structured interviews as a method of data collection with key stakeholders proved to be challenging. Some of the respondents, particularly in higher-ranking positions, had very little time to offer for interview.

\section{Conclusions}

Having effective corporate governance is very essential for all healthcare institutions. Implementations of accreditation programs has had a positive overall impact on hospitals and leads to enhanced quality of care provided at the hospitals, patient safety, and better health outcomes. Additionally, workforce shall be considered as a mediating factor between health system outcomes and governance mechanisms as well as rewarding of staff members was essential to overcome resistance. The findings showed that the corporate governance shown by the top management offered key participation that significantly contributed towards the implementation of accreditation and improvement in the hospital quality performance. All the hospital directors, executive leaders at the hospitals recognized the importance of their role and support in the accreditation process. They have given details of their participation at all of the different stages of the accreditation process: This support made a significant contribution to the improvement of the quality performance of the hospital as stated by the middle and line managers in addition to the employees. 


\section{Future Implications}

Future studies should emphasize on the development of a national accreditation program that is well structured and benchmarked with the international ones at the same time tailored based on the nature and culture of the systems at the UAE. Moreover, a robust monitoring system across the country and among the different facilities of MOHAP is needed to ensure the hospitals are achieving the standards, continuously implementing it and ensuring its sustainability along with helping employees measure and compare their own performance against standards which may lead to improvement behavior.

\section{Declarations}

\section{Acknowledgement}

We would like to acknowledge Ministry of Health and Prevention, Dubai, United Arab Emirates for the support.

\section{Ethics approval and consent to participate}

Individual participant consent and Institutional Review Board namely "Hamdan Bin Mohammed Smart University ( $\mathrm{HbMsU})$ and the Medical Research Ethics Committee at the Ministry of Health and Prevention (MOHAP) at the United Arab Emirates (UAE)" approval was sought and granted for this project. All participants provided written as well as verbal consent to take part in the study.

\section{Consent for publication}

Not applicable. The manuscript does not contain patient identifiable data.

\section{Funding}

None declared

\section{Competing interests}

The authors declare that they have no competing interests.

\section{Availability of data and materials}

The datasets analyzed/ generated during the current study are not publicly available due to patient confidentiality.

\section{References}

1. Devkaran S, O'Farrell PN. The impact of hospital accreditation on quality measures: an interrupted time series analysis. BMC health services research. 2015 Dec;15(1):137. 
2. Shaw, C. Toolkit for accreditation programmes. Some issues in the design and redesign of external health care assessment and improvement systems. International Society for Quality in Health Care. 2004.

3. Pomey MP, Lemieux-Charles L, Champagne F, Angus D, Shabah A, Contandriopoulos AP. Does accreditation stimulate change? A study of the impact of the accreditation process on Canadian healthcare organizations. Implementation Science. 2010 Dec;5(1):31.

4. Braithwaite J, Westbrook J, Pawsey M, Greenfield D, Naylor J, ledema R, Runciman B, Redman S, Jorm C, Robinson M, Nathan S. A prospective, multi-method, multi-disciplinary, multi-level, collaborative, social-organisational design for researching health sector accreditation [LP0560737]. BMC health services research. 2006 Dec;6(1):113.

5. Hinchcliff R, Greenfield D, Moldovan M, Westbrook JI, Pawsey M, Mumford V, Braithwaite J. Narrative synthesis of health service accreditation literature. BMJ Qual Saf. 2012 Dec 1;21(12):979-91.

6. Cret B. Accreditations as local management tools. Higher Education. 2011 Apr 1;61(4):415-29.

7. Greenfield D, Pawsey M, Braithwaite J. What motivates professionals to engage in the accreditation of healthcare organizations? International Journal for Quality in Health Care. 2010 Nov 16;23(1):814.

8. Braithwaite J, Westbrook J, Pawsey M, Greenfield D, Naylor J, ledema R, Runciman B, Redman S, Jorm C, Robinson M, Nathan S. A prospective, multi-method, multi-disciplinary, multi-level, collaborative, social-organisational design for researching health sector accreditation [LP0560737]. BMC health services research. 2006 Dec;6(1):113.

9. Nicklin W. The value and impact of accreditation in health care: a review of the literature. Accreditation Canada; 2009.

10. Chuang S, Inder K. An effectiveness analysis of healthcare systems using a systems theoretic approach. BMC Health Services Research. 2009 Dec;9(1):195.

11. Fukuyama F. What is governance? Governance. 2013; 26:347-368

12. Braithwaite J, Greenfield D, Westbrook J, Pawsey M, Westbrook M, Gibberd R, Naylor J, Nathan S, Robinson M, Runciman B, Jackson M. Health service accreditation as a predictor of clinical and organisational performance: a blinded, random, stratified study. BMJ Quality \& Safety. 2010 Feb 1;19(1):14-21.

13. Vision 2021 [Internet]. Dubai: UAE Prime Minister's Office; 2017. National Agenda Available at: https://www.vision2021.ae/en/national-priority-areas.

14. Schmaltz SP, Williams SC, Chassin MR, Loeb JM, Wachter RM. Hospital performance trends on national quality measures and the association with Joint Commission accreditation. Journal of hospital medicine. 2011 Oct;6(8):454-61.

15. El-Jardali F, Jamal D, Dimassi H, Ammar W, Tchaghchaghian V. The impact of hospital accreditation on quality of care: perception of Lebanese nurses. International Journal for Quality in Health Care. 2008 Jul 1;20(5):363-71. 
16. El-Jardali F, Hemadeh R, Jaafar M, Sagherian L, El-Skaff R, Mdeihly R, Jamal D, Ataya N. The impact of accreditation of primary healthcare centers: successes, challenges and policy implications as perceived by healthcare providers and directors in Lebanon. BMC health services research. 2014 Dec;14(1):86.

17. Alkhenizan A, Shaw $C$. The attitude of health care professionals towards accreditation: a systematic review of the literature. Journal of family \& community medicine. 2012 May;19(2):74.

18. Ongori H. A review of the literature on employee turnover. African Journal of Business Management; 49-54.

19. Richman AL, Civian JT, Shannon LL, Jeffrey Hill E, Brennan RT. The relationship of perceived flexibility, supportive work-life policies, and use of formal flexible arrangements and occasional flexibility to employee engagement and expected retention. Community, work and family. 2008 May 1;11(2):183-97. 